\title{
Application and Evolution for Neural Network and Signal Processing in Large-Scale Systems
}

\author{
Dongbao Jia, ${ }^{1,2}$ Cunhua Li, ${ }^{1}$ Qun Liu, ${ }^{3}$ Qin Yu, ${ }^{1}$ Xiangsheng Meng, ${ }^{4}$ Zhaoman Zhong $\mathbb{D}^{1}$, \\ Xinxin Ban $\mathbb{D}^{5},{ }^{5}$ and Nizhuan Wang $\mathbb{D}^{1}$ \\ ${ }^{1}$ School of Computer Engineering, Jiangsu Ocean University, Lianyungang 222005, China \\ ${ }^{2}$ MOE Key Laboratory of TianQin Project, Sun Yat-sen University, Guangzhou 510275, China \\ ${ }^{3}$ Tianwan Nuclear Power Plant, Lianyungang 222005, China \\ ${ }^{4}$ The First People's Hospital of Lianyungang, Lianyungang 222005, China \\ ${ }^{5}$ School of Environmental and Chemical Engineering, Jiangsu Ocean University, Lianyungang 222005, China \\ Correspondence should be addressed to Zhaoman Zhong; zhongzhaoman@163.com, Xinxin Ban; banxx@jou.edu.cn, and \\ Nizhuan Wang; wangnizhuan1120@gmail.com
}

Received 10 October 2020; Revised 18 December 2020; Accepted 7 March 2021; Published 10 April 2021

Academic Editor: Dan Selisteanu

Copyright (C) 2021 Dongbao Jia et al. This is an open access article distributed under the Creative Commons Attribution License, which permits unrestricted use, distribution, and reproduction in any medium, provided the original work is properly cited.

Low frequency oscillation is an important attribute of human brain activity, and the amplitude of low frequency fluctuation (ALFF) is an effective method to reflect the characteristics of low frequency oscillation, which has been widely used in the treatment of brain diseases and other fields. However, due to the low accuracy of the current analysis methods for low frequency signal extraction of ALFF, we propose the Fourier-based synchrosqueezing transform (FSST), which is often used in the field of signal processing to extract the ALFF of the low frequency power spectrum of the whole-time dimension. The low frequency characteristics of the extracted signal are compared with those of FSST and fast Fourier transform (FFT) through the resting-state data. It is clear that the signal extracted by FSST has more low frequency characteristics, which is significantly different from FFT.

\section{Introduction}

In recent years, with the rapid development of big data, especially artificial intelligence technology, intelligent methods have become increasingly popular in the operation and management optimization of complex large-scale systems, which often appear in ecological environments, communication networks, informatics, biology, and other disciplines. Extensive research has focused on medicine and health such as deep neural network, neuroscience, and brain science related purposes, data collection, accurate analysis, monitoring, and connecting available medical resources and healthcare services [1-6].

The brain is comprised of $10^{11}$ neurons and generally $10^{15}$ interconnections among them can handle massive information. Their shape and size are diverse, and their structures are distinct. The components of a neuron can be categorized as cell body, dendrite, and axon. Signals received from other neurons are reckoned into synapses and sent to the cell body. Once the signal entering the cell body surpasses the sustaining threshold, the neuron is burned, and the signal is transmitted to other neurons through axons [7].

The amplitude of low frequency fluctuation (ALFF) is a good measurement index of brain activation signal, which is calculated to obtain the power spectrum of brain voxel signal time series using time-frequency (TF) analysis method [8-11]. It is considered able to directly reflect the spontaneous synchronous changes of neural activity in a resting state $[12,13]$ and to some extent reflect the interaction and neural network connection among the relevant brain regions, which has been verified in the research of visual stimulus differences caused by eye opening and closing. Furthermore, ALFF can serve as a starting point for understanding brain diseases and can help reveal many pathological mechanisms, such as some mental diseases $[14,15]$. Therefore, improving the analytical accuracy of 
ALFF has a positive effect on the improvement of brain science research and the treatment of mental diseases [16-19].

Many signals such as audio signals and medical data (electrocardiogram and thoracic motion signals) can be modeled as superposition of amplitude-modulation and frequency-modulation (AM-FM) modes. Linear techniques such as wavelet transform and fast Fourier transform (FFT) are widely utilized to represent the characteristics of the signal in TF domain [20-24]. However, they all have the same limitation; that is, they cannot locate a signal with arbitrary accuracy in time and frequency. Even though an improved TF representation method called "reassignment" method (RM) was proposed, its main problem is that the reassigned transformation is no longer reversible and does not allow for the mode reconstruction. Moreover, it is difficult to achieve compatibility between the time and frequency resolutions because the frequency resolution depends on the length of the analysis window, and the analytical window length needs to be fixed according to the frequency. In particular, if the period of a signal is not constant, such as the sample signal for the object being relatively active, the analytical precision decreases $[25,26]$. Therefore, FFT cannot analyze the characteristics of the signal at a certain time accurately, resulting in a large amount of signal information losses, which is unsuitable for brain science analysis particularly.

In the analysis of audio signal [27, 28], another phasebased technique called "synchrosqueezing transform" (SST) was proposed. Its purpose is similar to that of RM, which is to sharpen the time-scale representation given by wavelet transform, and has the advantage of allowing for mode retrieval. Based on the principle of wavelet-based SST, an extension of SST to the TF representation given by shorttime Fourier transform called Fourier-based synchrosqueezing transform (FSST) was proposed [29], and it is proved that this method is robust to small bounded perturbations and noise [30]. Moreover, some researches proposed improving the existing FSST by using higher-order amplitude and phase approximation to calculate more accurate instantaneous frequency estimates of the modal components of the signal. It is achieving a perfect concentration and reconstruction in a wider range of AM-FM modes, and most of the real signals are composed of strongly modulated AM-FM modes, such as chirps involved in radar [31], speech processing [32], or gravitational waves $[9,11,33]$. As a consequence, this technique provides a highly concentrated TF representation for a wide variety of AM-FM multicomponent signals and enables the reconstruction of their modes with high accuracy; we hypothesized that FSST can be used in the analysis of time series of brain science and to improve the analytical accuracy.

In the present study, we propose and attempt the application of FSST to brain wave analysis and compare the results obtained by FFT and FSST analysis using data with no task resting state. Based on learning and improving the previous ALFF algorithm, the ALFF in the overall time domain is recalculated. According to the comparison with traditional ALFF values, we demonstrate that FSST is a highly precise method, which yields marked improvements in the reconstruction of TF signal. This technique is effective for analyzing and visualizing ALFF values and changes in brain waves.

\section{Materials and Methods}

The FFT as a Fourier-related transform has been commonly used for analyzing organic signals, such as the brain wave signal. The Fourier transform can be represented as follows:

$$
X(n f 1)=\int_{-\infty}^{\infty} x(t) e^{-\inf _{1} t} \mathrm{dt}
$$

where $t$ is the analysis window length $\left(f_{1}=1 / t, n\right.$ is a positive integer). Equation (1) is solved for determining the Fourier coefficients. As the Fourier transform is used for analyzing a completely periodic signal in an analysis window $t$, the calculated frequencies ( $n f 1)$ depend on the window length $t$, and errors frequently occur in the analysis of nonharmonic signal frequencies. Moreover, if changes in the frequency of the sample signal for the object are relatively active over a short duration, a decrease in the length of the analysis window to increase the time resolution also decreases the frequency resolution; the resulting analytical precision decreases.

As we all know, it is not appropriate to describe the nonstationary signal with time-domain or frequency-domain representation. Therefore, the short-time Fourier transform (STFT) is introduced and defined as follows:

$$
V_{f}^{g}(t, \eta)=\int_{R} f(\tau) g^{*}(\tau-t) e^{-2 i \pi \eta(\tau-t)} d \tau,
$$

where $g^{*}$ is the complex conjugate of $g$. Then, the spectrum corresponds to $\left|V_{f}^{g}(t, \eta)\right|^{2}$, and the original signal $f$ can be extracted from the STFT under the condition that $g$ does not disappear and is continuously at 0 :

$$
f(t)=\frac{1}{g^{*}(0)} \int_{R} V_{f}^{g}(t, \eta) d \eta .
$$

If $f$ is analytic, which means $\eta \leq 0$, then $\hat{f}(\eta)=0$; the integral in (3) only happens on $R_{+}$.

Synchrosqueezing transform can separate and adjust the signals of different modes by redistributing coefficients in series or frequency to avoid the uncertainty of linear transformation. It can analyze and compress the low frequency amplitude, obtain more accurate high-power signals, and find active points in brain regions. The signal can be expressed clearly while maintaining the reversibility of the signal.

The Fourier-based synchrosqueezing transform (FSST) technique is a newly proposed analytical method that has some advantages, as evidenced by the numerical processing of synthetic and observed gravitational wave signals [34, 35]. Time $t$ and frequency $\eta$ are predicted by sharpening the blurred STFT representation using the following instantaneous frequency of FSST: 
TABLE 1: Relevant information of echo planar imaging and fast gradient echo image.

\begin{tabular}{|c|c|c|c|c|c|c|c|c|}
\hline & Slices & $\begin{array}{l}\text { Thickness/ } \\
\text { gap }\end{array}$ & $\begin{array}{l}\text { In-plane } \\
\text { resolution }\end{array}$ & $\begin{array}{l}\text { Repetition time } \\
(\mathrm{ms})\end{array}$ & $\begin{array}{l}\text { Echo time } \\
(\mathrm{ms})\end{array}$ & Field of view & $\begin{array}{l}\text { Turning } \\
\text { angle }\end{array}$ & $\begin{array}{l}\text { Inversion } \\
\text { time }\end{array}$ \\
\hline $\begin{array}{l}\text { Echo planar } \\
\text { imaging }\end{array}$ & 33 axial & $3.5 / 0.7 \mathrm{~mm}$ & $64^{*} 64$ & 2000 & 30 & $200^{*} 200 \mathrm{~mm}^{2}$ & $90^{\circ}$ & - \\
\hline $\begin{array}{l}\text { Fast gradient echo } \\
\text { image }\end{array}$ & $\begin{array}{c}128 \\
\text { sagittal }\end{array}$ & $1.33 / 0 \mathrm{~mm}$ & $256^{*} 192$ & 2530 & 3.39 & $256^{*} 256 \mathrm{~mm}^{2}$ & $7^{\circ}$ & $1100 \mathrm{~ms}$ \\
\hline
\end{tabular}

$$
\widehat{\omega_{f}}(t, \eta)=\frac{1}{2 \pi} \partial_{t} \arg \left\{V_{f}^{g}(t, \eta)\right\},
$$

where $\partial_{\mathrm{t}}$ is the partial derivative with respect to $t$. Indeed, $V_{f}^{g}(t, \eta)$ is redistributed to a new location $\left(t, \widehat{\omega_{f}}(t, \eta)\right)$ using the following synchrosqueezing operator:

$$
T_{f}^{g, \gamma}(t, \omega)=\frac{1}{g^{*}(0)} \int_{\left\{\eta,\left|V_{f}^{g}(t, \eta)\right|>\gamma\right\}} V_{f}^{g}(t, \eta) \delta\left(\omega-\widehat{\omega}_{f}(t, \eta)\right) d \eta,
$$

where $\gamma$ is the threshold. By integrating $T_{f}^{g, \gamma}(t, \eta)$ near the corresponding ridge $\left(t, \phi_{k}^{\prime}(t)\right)$, the $k^{\text {th }}$ mode is reconstructed approximately, since the coefficients are redistributed along the frequency axis, and FSST maintains the nature of causality:

$$
f_{k}(t) \approx \int_{\left\{\omega,\left|\omega-\varphi_{k}(t)\right|<d\right\}} T_{f}^{g, \gamma}(t, \omega) d \omega,
$$

where $\varphi_{k}(t)$ is an estimate of $\phi_{k}^{\prime}(t)$ and the parameter $d$ enables compensating for the imprecise approximation $\varphi_{k}(t)$ of $\phi_{k}^{\prime}(t)$ and the error made by estimating the IF by means of $\widehat{\omega_{f}}(t, \eta)$. In addition, the approximation $\varphi_{k}(t)$ must be calculated before retrieving mode $f_{k}$. As a consequence, a commonly used technique that is based on ridge extraction predicting $T_{f}^{g, \gamma}$ and $K$ is known [33]. Therefore, FSST as a highly precise frequency analysis method can be used to analyze brain waves and improve the analytical precision in the present study.

2.1. Experiment. Forty-seven participants in their $20 \mathrm{~s}-40 \mathrm{~s}$ with no mental illness history participated in the present study of resting state. All participants provided written consent to participate, and ethical approval was obtained from the institutional ethics committee [36, 37]. For avoiding the influence of outside equipment, the experiment was conducted in an anechoic chamber with lights off.

In functional imaging, echo planar imaging and fast gradient echo image were obtained by three-dimensional T1-weighted magnetization with the relevant information shown in Table 1, respectively [38-40].

All of forty-seven participants' closed-eye sample data were used in the resting experiment. Each condition consists of 240 functional volumes; in terms of data selection, we discard the original 10 point volumes and reserve 120 volumes.

The improvement of the algorithm is mainly reflected in the calculation and selection of power spectrum; in terms of analytical conditions, as the brain activity is the most obvious and active in this piece of data, FFT and FSST both select the average value in the time series with the frequency band of $0.01-0.1 \mathrm{~Hz}$ and the data length of $20-420 \mathrm{~s}$ in the total length of 9 minutes. The analysis window length is $2^{*} \mathrm{ABS}$ (data)/sample length. Furthermore, FFT and FSST algorithms are used to extract ALFF values and conduct comparative analysis; the coefficient values of the corresponding ALFF are extracted as follows (Table 2).

\section{Results and Discussion}

The experimental data of each participant were extracted twice. We divided these two groups of data into Test1 and Test2; FFT and FSST are used to analyze the two groups of data. Results of participant 32 as an example are shown in Figure 1 since it is nonspecific, which are Test1 FSST, Test1 FFT, Test2 FSST, and Test2 FFT, respectively. Each group of images shows 12 cross sections of the brain, and the corresponding position of each cross section is shown in the longitudinal view of brain area in the lower right corner of each group of images.

Although the high amplitude of FSST and FFT is only about 3000 in the resting state, the difference between FSST and FFT is obvious. From the point of view of images, FSST detects the results that cannot be resolved by FFT and their mean coefficients, which proves that FSST is more accurate and sensitive than FFT in data analysis and image extraction and highlighting. In particular, FSST highlights the superior frontal fornix, rectus, and other areas, which are valuable for the scientific research of brain activity and mental disease. Therefore, the difference between FSST and FFT is large, whose accuracy is also higher.

Since the amplitude of the low frequency band is used in this experiment, the average sum of these values should be carried out in FFT analysis of these multiple detection points, and then the frequency power spectrum is obtained by judging the ratio of the low frequency band (0.01-0.1 Hz) to each frequency band; the obtained frequency power spectrum is analyzed to determine whether these points conform to the low frequency amplitude attribute. To further analyze the significance of these signals, we introduce the mean correlation coefficient to correlate the extracted signals and calculate the average value, as shown in the figure below.

We can clearly see that, in both the mean coefficient and the low frequency ratio of the two groups of data, the multiple detection parts of FSST are indeed better than those 
TABLE 2: Coefficient values of ALFF for FFT and FSST in resting-state experiment.

\begin{tabular}{|c|c|c|c|c|c|}
\hline Participant & FFT & FSST & Participant & FFT & FSST \\
\hline 1 & 0.85 & 0.81 & 25 & 0.85 & 0.90 \\
\hline 2 & 0.92 & 0.87 & 26 & 0.89 & 0.82 \\
\hline 3 & 0.94 & 0.90 & 27 & 0.89 & 0.85 \\
\hline 4 & 0.96 & 0.91 & 28 & 0.90 & 0.89 \\
\hline 5 & 0.91 & 0.86 & 29 & 0.90 & 0.82 \\
\hline 6 & 0.95 & 0.91 & 30 & 0.95 & 0.89 \\
\hline 7 & 0.92 & 0.88 & 31 & 0.93 & 0.94 \\
\hline 8 & 0.84 & 0.74 & 32 & 0.87 & 0.77 \\
\hline 9 & 0.87 & 0.85 & 33 & 0.52 & 0.47 \\
\hline 10 & 0.94 & 0.93 & 34 & 0.84 & 0.80 \\
\hline 11 & 0.82 & 0.75 & 35 & 0.91 & 0.86 \\
\hline 12 & 0.95 & 0.92 & 36 & 0.86 & 0.74 \\
\hline 13 & 0.81 & 0.85 & 37 & 0.92 & 0.89 \\
\hline 14 & 0.91 & 0.89 & 38 & 0.90 & 0.87 \\
\hline 15 & 0.94 & 0.91 & 39 & 0.93 & 0.93 \\
\hline 16 & 0.86 & 0.70 & 40 & 0.92 & 0.91 \\
\hline 17 & 0.87 & 0.78 & 41 & 0.79 & 0.70 \\
\hline 18 & 0.95 & 0.92 & 42 & 0.88 & 0.83 \\
\hline 19 & 0.92 & 0.90 & 43 & 0.90 & 0.87 \\
\hline 20 & 0.94 & 0.87 & 44 & 0.92 & 0.92 \\
\hline 21 & 0.94 & 0.90 & 45 & 0.95 & 0.93 \\
\hline 22 & 0.97 & 0.95 & 46 & 0.93 & 0.92 \\
\hline 23 & 0.90 & 0.89 & 47 & 0.96 & 0.93 \\
\hline 24 & 0.95 & 0.94 & Mean & 0.90 & 0.86 \\
\hline
\end{tabular}

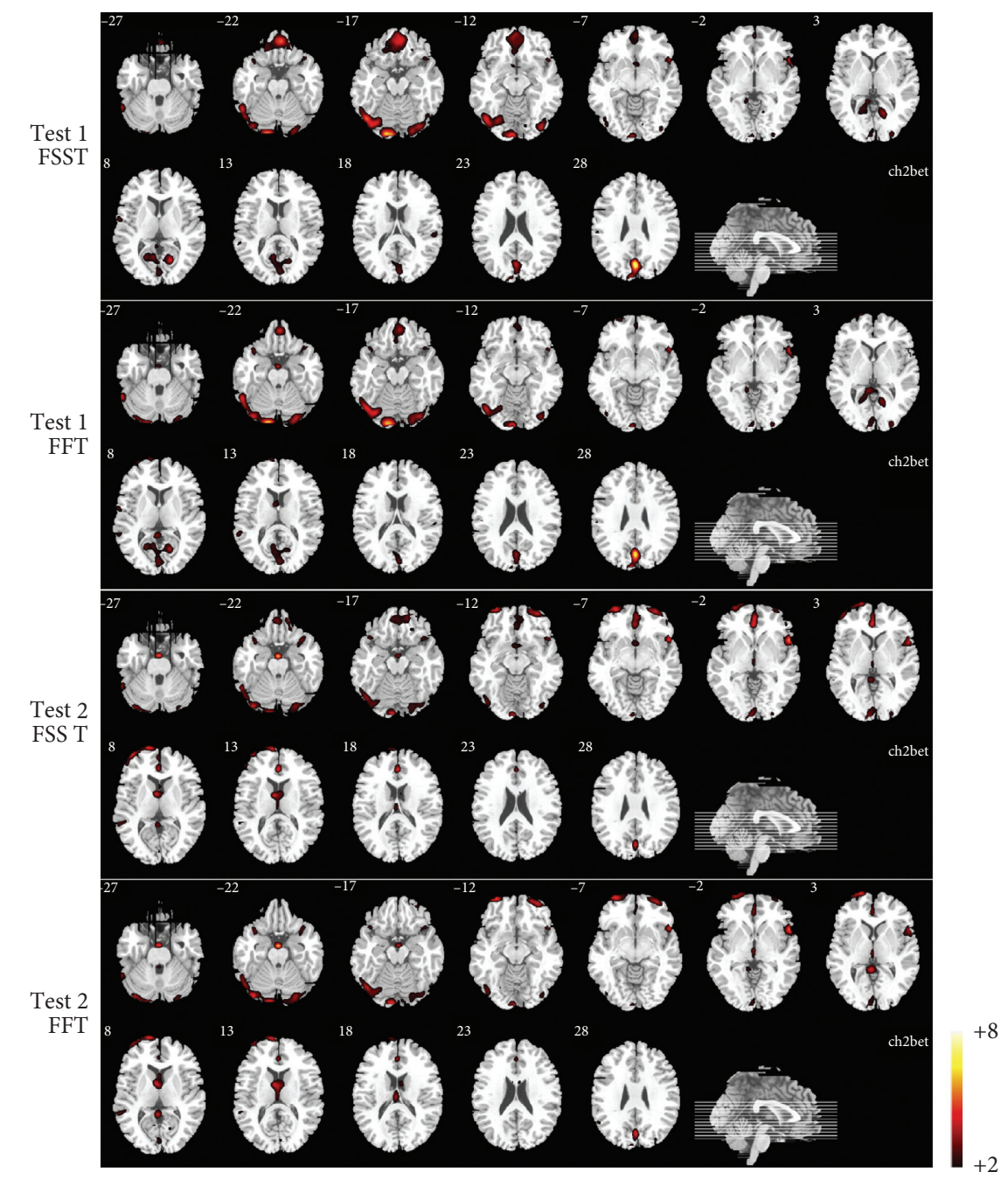

FIgURE 1: Analysis results of 2 groups of data from participant 33 using FSST and FFT technique. 


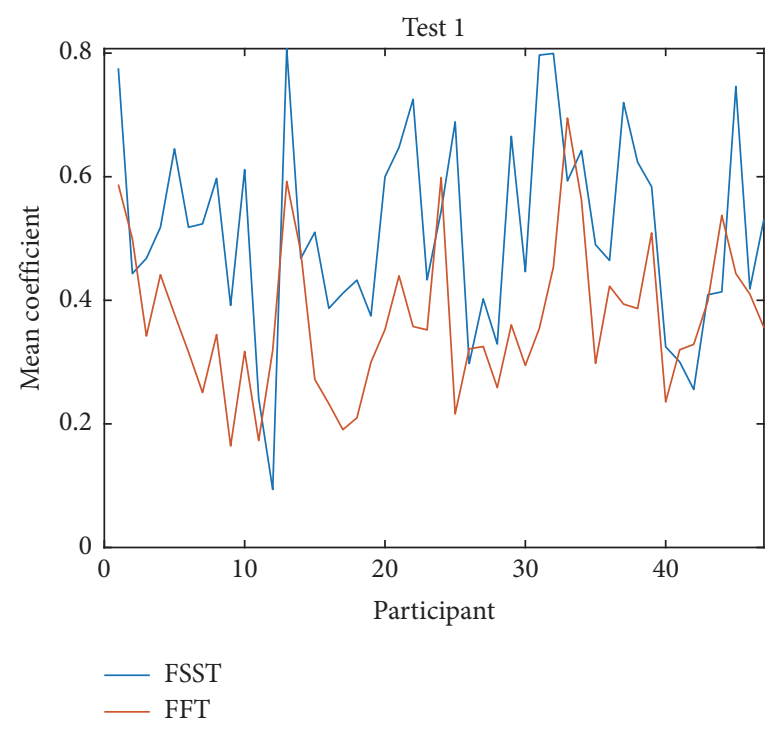

(a)

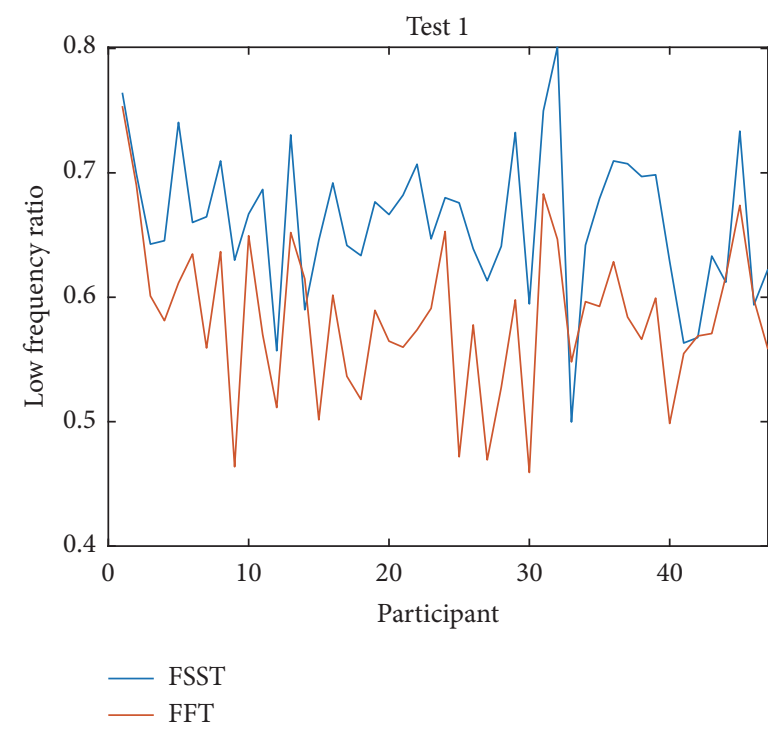

(c)

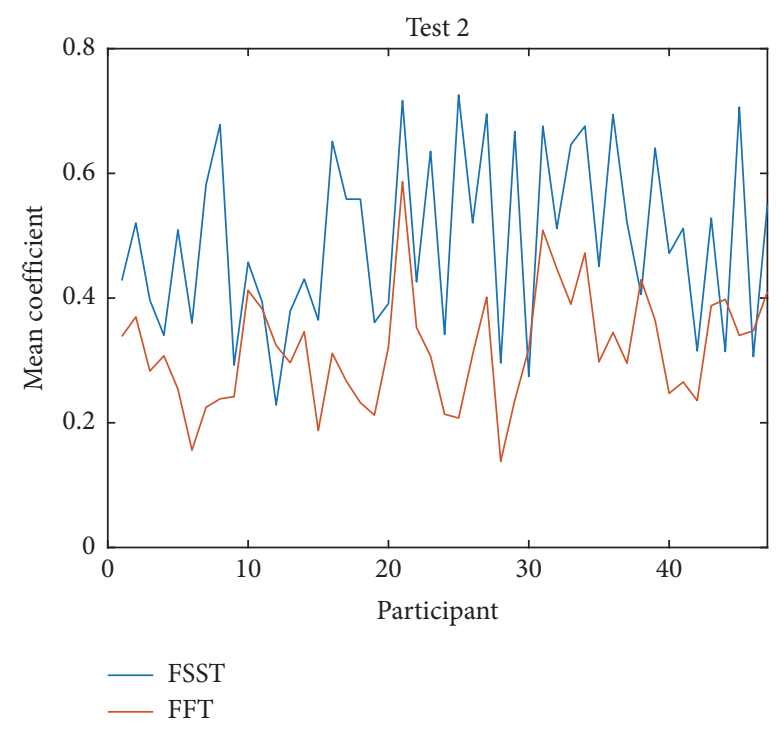

(b)

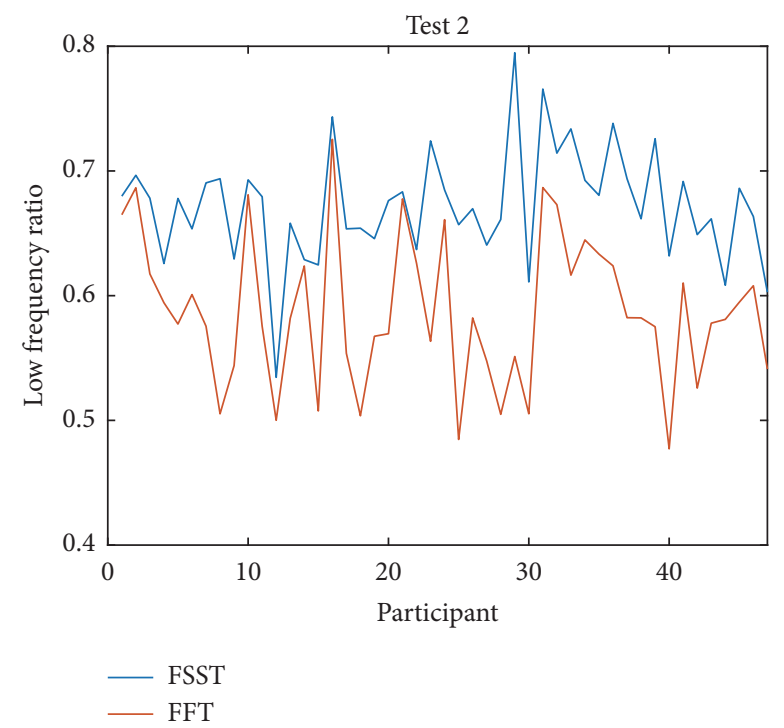

(d)

FIgURE 2: Mean coefficient and low frequency ratio of each participant in low frequency band using FSST and FFT.

of FFT, as shown in Figure 2. Since the significance of the low frequency coefficient can reflect the significance of the ALFF index and the difference of the low frequency amplitude is the reason for the difference of the low frequency coefficient, the low frequency amplitude value targeted by this experiment can show the characteristics of ALFF. Therefore, it is also proved that FSST is clearly superior to FFT in the analysis of ALFF value, which has stronger low frequency characteristics and more obvious ability to extract low frequency characteristics.

Figure 3 shows the area with $P$ value less than 0.05 after the examination. Set 18 adjacent voxels (edge connection, the default value of SPM) and voxel number of 200 as the cluster size parameter, perform cluster reading on these regions, and the region with voxel greater than 70 is selected from the cluster for display, since the number of regions is too large. The positive value is the region with significant difference between FSST and FFT. Negative values represent areas where there is a significant difference between FFT and FSST. As shown in Figure 2, the FSST and FFT also show significant differences in Figure 3.

Based on the analysis and the performance of the actual effect, the significance of the algorithm improvement is proved. The performance of ALFF index has been greatly improved which promotes the application of ALFF and its value. 


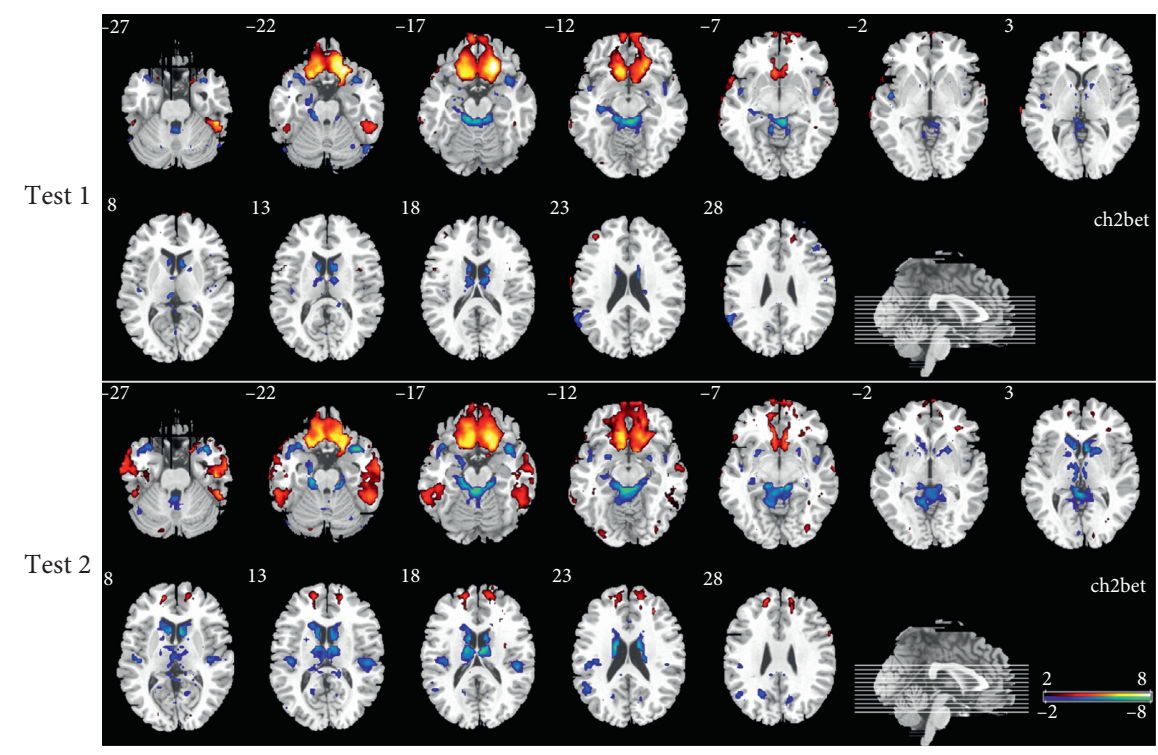

FIgURE 3: Difference results of FSST and FFT in Test1 and Test2.

\section{Conclusions}

At present, the research on human brain occupational plasticity has been developed rapidly, but the research on ALFF needs to be further improved. Because of the complexity and variety of EEG signals, extracting the information of the signals in time series can effectively reduce the signal ignorance caused by frequency band concentration.

Based on the previous research, this paper proposes the FSST algorithm to improve the resolution and enhance the analytical accuracy of ALFF, aiming to improve the visualization effect of ALFF and the extraction effect of low frequency signals. From the experimental results, it is proved that there is a significant difference between the overall analytical effects of FSST and FFT in resting state, and the extraction precision of FSST is significantly higher, which can reflect the TF structure characteristics of brain waves clearly.

In addition, FSST can detect the changes of brain dynamics in time series. After the processing, the TF map of the signal can be recovered to a greater extent. Based on the accuracy of the recovered signal and the characteristics of low frequency attributes, it is proved that FSST algorithm is feasible for the detection and analysis of ALFF in the time domain. In the future, we will verify the applicability of FSST to ALFF in task-related experiments.

\section{Data Availability}

The data used to support the findings of this study are available from the corresponding author upon request.

\section{Conflicts of Interest}

The authors declare that there are no conflicts of interest regarding the publication of this paper.

\section{Acknowledgments}

This research was supported by the Natural Science Foundation of the Jiangsu Higher Education Institutions of China under Grants 19KJB160001 and 19KJB520004, the Lianyungang city Haiyan project under Grant 2019-QD-004, the MOE Key Laboratory of TianQin Project, Sun Yat-sen University, and the Open Fund Project of Jiangsu Institute of Marine Resources Development under Grant JSIMR202018. The authors would like to thank Qin Yu for his contribution at the early phase of this work.

\section{References}

[1] R. Xu, N. Jiang, C. Lin et al., "Enhanced low-latency detection of motor intention from EEG for closed-loop brain-computer interface application," IEEE Transactions on Biomedical Engineering, vol. 61, no. 2, pp. 288-296, 2014.

[2] J. Kevric and A. Subasi, "Comparison of signal decomposition methods in classification of EEG signals for motor-imagery BCI system," Biomedical Signal Processing and Control, vol. 31, pp. 398-406, 2017.

[3] S. A. Sprague, M. T. McBee, and E. W. Sellers, "The effects of working memory on brain-computer interface performance," Clinical Neurophysiology, vol. 127, no. 2, pp. 1331-1341, 2016.

[4] S. P. Levine, J. E. Huggins, S. L. BeMent et al., "A direct brain interface based on event-related potentials," IEEE Transactions on Rehabilitation Engineering, vol. 8, no. 2, pp. 180-185, 2000.

[5] S. Gao, Y. Yu, Y. Wang, J. Wang, J. Cheng, and M. Zhou, "Chaotic local search-based differential evolution algorithms for optimization," IEEE Transactions on Systems, Man, and Cybernetics: Systems, p. 1. In press, 2020.

[6] J. Li, X. J. Duan, Q. Cui et al., "More than just statics: temporal dynamics of intrinsic brain activity predicts the suicidal ideation in depressed patients," Psychological Medicine, vol. 49, no. 5, pp. 1-9, 2018.

[7] S. Gao, M. Zhou, Y. Wang, J Cheng, H Yachi, and J Wang, "Dendritic neuron model with effective learning algorithms 
for classification, approximation, and prediction," IEEE Transactions on Neural Networks and Learning Systems, vol. 30, no. 2, pp. 601-614, 2019.

[8] J. Sun, S. Gao, H. Dai, J. Cheng, M. Zhou, and J. Wang, "Biobjective elite differential evolution algorithm for multivalued logic networks," IEEE Transactions on Cybernetics, vol. 50, no. 1, pp. 233-246, 2020.

[9] D. Jia, K. Yanagisawa, Y. Ono et al., "Multiwindow nonharmonic analysis method for gravitational waves," IEEE Access, vol. 6, pp. 48645-48655, 2018.

[10] L. Yang, Y. Yan, Y. H. Wang et al., "Gradual disturbances of the amplitude of low-frequency fluctuations (ALFF) and fractional ALFF in Alzheimer spectrum," Frontiers in Neuroscience, vol. 12975 pages, 2018.

[11] D. Jia, K. Yanagisawa, M. Hasegawa et al., "Time-frequencybased non-harmonic analysis to reduce line-noise impact for LIGO observation system," Astronomy and Computing, vol. 25, pp. 238-246, 2018.

[12] Y. R. Wang, S. C. Gao, M. C. Zhou et al., "A multi-layered gravitational search algorithm for function optimization and real-world problems," IEEE/CAA Journal of Automatica Sinica, In press, 2020.

[13] D. Jia, H. Dai, Y. Takashima et al., "EEG processing in internet of medical things using non-harmonic analysis: application and evolution for SSVEP responses," IEEE Access, vol. 7, pp. 11318-11327, 2019.

[14] Q. Cui, W. Sheng, Y. Chen et al., "Dynamic changes of amplitude of low-frequency fluctuations in patients with generalized anxiety disorder," Human Brain Mapping, vol. 41, no. 6, pp. 1667-1676, 2020.

[15] W. Liao, H. Chen, J. Li et al., "Endless fluctuations: temporal dynamics of the amplitude of low frequency fluctuations," IEEE Transactions on Medical Imaging, vol. 38, no. 11, pp. 2523-2532, 2019.

[16] G. Rodríguez-Bermúdez, P. J. García-Laencina, J. RocaGonzález, and J. Roca-Dorda, "Efficient feature selection and linear discrimination of EEG signals," Neurocomputing, vol. 115, pp. 161-165, 2013.

[17] J. Atkinson and D. Campos, "Improving BCI-based emotion recognition by combining EEG feature selection and kernel classifiers," Expert Systems with Applications, vol. 47, pp. 35-41, 2016.

[18] Linux.com. Who needs the internet of things? https://www. linux.com/news/who-needs-internetthings.

[19] D. B. Jia, Y. Fujishita, C. H. Li et al., "Validation of large-scale classification problem in dendritic neuron model using particle antagonism mechanism," Electronics, vol. 9, no. 5, 2020.

[20] H. Shen, L. Zhao, Y. Bian, and L. Xiao, "Research on SSVEPbased controlling system of multi-DoF manipulator," Advances in Neural Networks-ISNN 2009, Springer, Berlin, Germany, pp. 171-177, 2009.

[21] Z. Iscan and V. V. Nikulin, "Steady state visual evoked potential (SSVEP) based brain-computer interface (BCI) performance under different perturbations," PLoS One, vol. 13, no. 1, Article ID e0191673, 2018.

[22] A. M. Dreyer and C. S. Herrmann, "Frequency-modulated steady-state visual evoked potentials: a new stimulation method for brain-computer interfaces," Journal of Neuroscience Methods, vol. 241, pp. 1-9, 2015.

[23] J. Artieda, M. Valencia, M. Alegre, O. Olaziregi, E. Urrestarazu, and J. Iriarte, "Potentials evoked by chirpmodulated tones: a new technique to evaluate oscillatory activity in the auditory pathway," Clinical Neurophysiology, vol. 115, no. 3, pp. 699-709, 2004.

[24] R. Ku, A. Duszyk, P. Milanowski et al., "On the quantification of SSVEP frequency responses in human EEG in realistic BCI conditions," PLoS One, vol. 8, no. 10, Article ID e77536, 2013.

[25] C.-M. Huang, Y.-F. Chen, S. Xu, and H. Zhou, "The vehicular social network (VSN)-Based sharing of downloaded geo data using the credit-based clustering scheme," IEEE Access, vol. 6, pp. 58254-58271, 2018.

[26] D. J. A. McKechan, C. Robinson, and B. S. Sathyaprakash, "A tapering window for time-domain templates and simulated signals in the detection of gravitational waves from coalescing compact binaries," Classical Quantum Gravity, vol. 27, no. 8, Article ID 084020, 2010.

[27] I. Daubechies and S. Maes, "A nonlinear squeezing of the continuous wavelet transform based on auditory nerve models," Wavelets in Medicine and Biology, pp. 527-546, Routledge, Abingdon, UK, 1996.

[28] I. Daubechies, J. Lu, and H.-T. Wu, "Synchrosqueezed wavelet transforms: an empirical mode decomposition-like tool," Applied and Computational Harmonic Analysis, vol. 30, no. 2, pp. 243-261, 2011.

[29] G. Thakur and H.-T. Wu, "Synchrosqueezing-based recovery of instantaneous frequency from nonuniform samples," SIAM Journal on Mathematical Analysis, vol. 43, no. 5, pp. 20782095, 2011.

[30] G. Thakur, E. Brevdo, N. S. Fučkar, and H.-T. Wu, "The synchrosqueezing algorithm for time-varying spectral analysis: robustness properties and new paleoclimate applications," Signal Processing, vol. 93, no. 5, pp. 1079-1094, 2013.

[31] M. Skolnik, Radar Handbook, Technology and Engineering, McGraw-Hill Education, New York, NY, USA, 2008.

[32] J. W. Pitton, L. E. Atlas, and P. J. Loughlin, "Applications of positive time-frequency distributions to speech processing," IEEE Transactions on Speech and Audio Processing, vol. 2, no. 4, pp. 554-566, 1994.

[33] D.-H. Pham and S. Meignen, "High-order synchrosqueezing transform for multicomponent signals analysis-with an application to gravitational-wave signal," IEEE Transactions on Signal Processing, vol. 65, no. 12, pp. 3168-3178, 2017.

[34] S. Klimenko and G. Mitselmakher, "A wavelet method for detection of gravitational wave bursts," Classical and Quantum Gravity, vol. 21, no. 20, pp. S1819-S1830, 2004.

[35] B. P. Abbott, R. Abbott, T. D. Abbott et al., "Observation of gravitational waves from a binary black hole merger," Physical Review Letters, vol. 116, no. 6, Article ID 061102, 2016.

[36] Ethics Committee, University of Jiangsu, "Ethical review of research for non-medical purposes," University of Jiangsu, Zhenjiang, China, 2020, https://kjc.ujs.edu.cn/info/1149/ 2963.htm

[37] G. Garcia, "High frequency SSVEPs for BCI Applications," in Proceedings of the Brain Computer Interfaces HCI Games Workshop, Florence, Italy, January 2008.

[38] D. Liu, Z. Dong, X. Zuo, J. Wang, and Y. Zang, "Eyes-open/ eyes-closed dataset sharing for reproducibility evaluation of resting state fMRI data analysis methods," Neuroinformatics, vol. 11, no. 4, pp. 469-476, 2013.

[39] A. Dutta, S. McKie, and J. F. W. Deakin, "Resting state networks in major depressive disorder," Psychiatry Research: Neuroimaging, vol. 224, no. 3, pp. 139-151, 2014

[40] C.-G. Yan, X.-D. Wang, X.-N. Zuo, and Y.-F. Zang, "DPABI: data processing \& analysis for (Resting-State) brain imaging," Neuroinformatics, vol. 14, no. 3, pp. 339-351, 2016. 